\title{
Dilemas na pesquisa induzida: experiência do Instituto Butantan no Programa de Pesquisa para o SUS
}

\author{
Dilemmas in induced research: experience of the Butantan Institute \\ in the Research Program for the SUS
}

Dilemas en la investigación inducida: experiencia del Instituto Butantan en el Programa de Investigación para el SUS

\begin{abstract}
Cristiano Corrêa de Azevedo Marques ${ }^{1(*)}$, Paulo Henrique Nico Monteiro², Suzane Eulália de Castro Jardim ${ }^{3}$, Mateus Lopes Teixeira ${ }^{4}$, Olga Sofia Fabergé Alves ${ }^{5}$

${ }^{1}$ Doutor em Ciências da Saúde, Pesquisador Científico do Laboratório Especial de História da Ciência do Centro de Desenvolvimento Cultural do Instituto Butantan - SES-SP

${ }^{2}$ Doutor em Educação pela USP, Pós Doutor em Comunicação Científica pela Laurentian University, Canadá, Pesquisador Científico do Laboratório Especial de História da Ciência do Centro de Desenvolvimento Cultural do Instituto Butantan - SES-SP

${ }^{3}$ Graduada em História pela Universidade de São Paulo

${ }^{4}$ Graduando em História pela USP, aluno de Iniciação Científica do Instituto Butantan - SES-SP

${ }^{5}$ Mestre em Ciências pela USP, Pesquisadora Científica do Laboratório Especial de História da Ciência do Centro de Desenvolvimento Cultural do Instituto Butantan - SES-SP
\end{abstract}

\section{RESUMO}

Este estudo analisa o perfil das instituições do estado de São Paulo contempladas pelos editais do Programa de Pesquisa para o Sistema Único de Saúde (PPSUS), comparativamente ao Instituto Butantan. Foram empregados métodos mistos de análise, utilizando bases de dados da FAPESP e CNPq, pesquisa documental e entrevistas com 34 líderes de grupos de pesquisa do Butantan cadastrados no CNPq em 2013. Identificamos 205 projetos financiados pelo PPSUS em São Paulo entre 2004 e 2012, período em que o Instituto Butantan aparece indiretamente como instituição proponente em apenas três projetos contemplados. Os entrevistados apontam a FAPESP como sua principal fonte de financiamento e consideram que os temas apresentados no PPSUS não se adequam às linhas de pesquisa institucional.

Autor de Correspondência:

${ }^{*}$ Cristiano Corrêa de Azevedo Marques. E-mail: cristiano.marques@butantan.gov.br 
Considerando o papel indutor do PPSUS à agenda de saúde do estado, e a capacidade científica instalada no Butantan, cabe o desafio de diminuir a distância entre essas duas agendas de pesquisa.

Palavras-chave: Financiamento Governamental. Academias e Institutos. Pesquisa sobre serviços de Saúde.

\begin{abstract}
This study analyzes the profile of the institutions of the state of São Paulo contemplated by the calls for proposals of the Research Program for the Unified Health System (PPSUS), compared to the Butantan Institute. We used mixed methods of analysis, using FAPESP and CNPq databases, documentary research, and interviews with 34 leaders of Butantan research groups registered at CNPq in 2013. There were 205 projects identified as sponsored by PPSUS in São Paulo between 2004 and 2012, period in which the Butantan Institute appears indirectly as a proposing institution in only three contemplated projects. The interviewees point to FAPESP as their main funding source and consider that the themes presented in the PPSUS do not fit the institutional research lines. Considering the role of PPSUS in the health agenda of the state, and the scientific capacity installed in Butantan, the challenge is to reduce the distance between these two research agendas.
\end{abstract}

Keywords: Financing, Government. Academies and Institutes. Health Services Research.

\title{
RESUMEN
}

Este estudio analizó el perfil de las instituciones del estado de São Paulo contempladas por las convocatorias del Programa de Investigación para el Sistema Único de Salud (PPSUS), en comparación con el Instituto Butantan. Se aplicaron métodos mixtos utilizando bases de datos de FAPESP y CNPq, investigación documental y entrevistas con 34 líderes de grupos de investigación de Butantan registrados en CNPq en 2013. Identificamos 205 proyectos financiados por el PPSUS en São Paulo entre 2004 y 2012, donde Instituto Butantan aparece indirectamente como proponente en tres proyectos contemplados. Los entrevistados apuntan FAPESP como su principal fuente de financiamiento y consideran que los temas presentados en PPSUS no se adecuan a las líneas de investigación institucional. Considerando el papel inductor de PPSUS a la agenda de salud del estado, y la capacidad científica instalada en el Butantan, cabe disminuir la distancia entre esas agendas de investigación.

Palabras clave: Financiación Gubernamental. Academias e Institutos; Investigación en Servicios de Salud.

\section{INTRODUÇÃ̃o}

A pesquisa em saúde no Brasil, desde o final do século XX, tem crescido em importância tornando-se objeto de políticas públicas específicas de financiamento e indução direcionada ao Sistema Único de Saúde (SUS). Os motivos desse crescimento estão associados ao contexto que envolve o desenvolvimento do sistema de saúde brasileiro, com decorrente aumento da demanda por soluções tecnológicas e otimização dos investimentos, aliado à transição em saúde, seja pelo envelhecimento da população, seja pelo surgimento 
de novas doenças transmissíveis, as quais demandam novos instrumentos e ferramentas de intervenção eficazes ${ }^{1,2}$.

Uma das causas deste crescimento pode ser atribuída à institucionalização da pesquisa em saúde no SUS, ocorrida no final dos anos 1990, a partir da realização das conferências nacionais de ciência e tecnologia em saúde, criação do Departamento de Ciência e Tecnologia do Ministério da Saúde (DECIT), estabelecimento da Política Nacional de Ciência e Tecnologia e Inovação em Saúde (PNCTIS) e da Agenda Nacional de Prioridades de Pesquisa em Saúde (ANPPS) ${ }^{3}$. Este processo resultou em um aumento dos componentes verticais de fomento à pesquisa em saúde, com ênfase na capacidade de indução, particularmente na perspectiva de tecnologia e inovação, como também no reforço ao componente empresarial, esse último decorrente de um entendimento da saúde, tanto como um resultado, quanto um fator de desenvolvimento econômico ${ }^{1,2}$.

Como decorrência deste movimento, em 2001, o Ministério da Saúde dá início a ações de indução de pesquisa em sete estados brasileiros. Em 2003 o programa é ampliado incluindo mais três estados e finalmente em 2004, com assinatura de convênios com as Fundações Estaduais de Apoio à Pesquisa (FAP's), estabelece o Programa de Pesquisa para o SUS (PPSUS), com os objetivos de apoiar financeiramente o desenvolvimento de pesquisas que busquem contribuir para a resolução de problemas prioritários de saúde da população brasileira, reduzir as desigualdades regionais de capacidade em pesquisa e fortalecer a gestão do SUS. Adicionalmente, este Programa prevê a execução descentralizada por meio das FAP's e estabelece uma metodologia de execução participativa, fundamentada na análise da situação de saúde dos níveis locais e regionais para seleção das prioridades em pesquisa a serem incorporados aos editais4. No Período de 2002-2012 foram investidos no país aproximadamente 275 milhões de reais, e o estado de São Paulo tem participado do programa, tornando-se o segundo maior investimento, abaixo apenas do estado de Minas Gerais - R\$34.120.000,00 e $R \$ 45.804 .000,00$ respectivamente. Esta diferença se dá principalmente pelo valor investido em Minas Gerais por meio de sua Secretaria Estadual de Saúde nos anos de 2006, 2008 e $2011^{5}$.

Neste contexto, é fundamental considerar que a partir de 2004 o estado de São Paulo adotou a prática de estabelecer Planos Estaduais de Saúde (PES) de caráter quadrienal que incorporam um eixo temático específico de Ciência, Tecnologia e Inovação em Saúde. No processo de construção do PES, que envolve a participação dos serviços de saúde, sociedade civil e academia, já se esboçam os principais aspectos e demandas para o SUS-SP. No eixo "ciência e tecnologia" do PES, estes elementos são considerados facilitadores do processo de construção de uma agenda de prioridades em pesquisa no estado e para o delineamento das prioridades de fomento descritas nos editais do PPSUS-SP ${ }^{6,7}$. A despeito de este programa ter início em 2001, o estado de São Paulo adere ao PPSUS a partir de 2004 adotando, ao longo do período, diferentes modalidades de construção das prioridades de pesquisa para os editais ${ }^{8}$.

Ainda integrando este contexto, a Secretaria da Saúde do Estado de São Paulo (SES-SP), possui um expressivo parque de C \& T composto de hospitais, unidades de referência técnica e científica, além de sete institutos de pesquisa, o que torna a SES-SP um ambiente relevante e oportuno para implementação e desenvolvimento de projetos de pesquisa financiados pelo PPSUS. Dentre os institutos de pesquisa da SES-SP, escolhemos como objeto de análise o Instituto Butantan, uma instituição centenária voltada à pesquisa, ensino, produção e difusão, cujo principal objeto é a pesquisa biomédica para a saúde pública, especialmente no que concerne a animais peçonhentos e produção de soros e vacinas. O objetivo deste estudo consiste em descrever o perfil da implementação do PPSUS no estado de São Paulo no período de 2004-2012 e analisar a participação do 
Instituto Butantan - entendido como lócus potencial para o desenvolvimento de pesquisas financiadas pelo PPSUS-SP - considerando as instituições contempladas pelo programa em relação a outras unidades e institutos da SES-SP e demais instituições de pesquisa do estado de São Paulo.

\section{MÉTODO}

Para esse estudo de natureza descritiva foram empregados métodos mistos ${ }^{9}$ utilizando duas fontes de dados.

Primeiramente foi realizada pesquisa documental em documentos de referência e nas seguintes bases de públicas de dados: Diretório de Grupos de Pesquisa cadastrados na Plataforma Lattes do Conselho Nacional de Desenvolvimento Científico e Tecnológico (CNPq); Portal da Fundação de Amparo à Pesquisa do Estado de São Paulo - (FAPESP $)^{10}$ e do Departamento de Ciência e Tecnologia do Ministério da Saúde - DECIT ${ }^{5}$. Devido a discrepâncias de informações verificadas entre as bases de dados do MS - DECIT e FAPESP, em especial relacionadas à periodicidade de vigência dos projetos, adotamos como referencial esta última fonte. As instituições participantes foram identificadas por meio dos dados cadastrais e vínculo institucional do pesquisador principal e beneficiário. Para análise quantitativa dos dados foi utilizada estatística descritiva utilizando Microsoft Excel ${ }^{\mathrm{TM}}$.

Como segunda fonte de dados, foram coletados dados primários por meio de entrevistas com pesquisadores do Instituto Butantan cadastrados como líderes de grupos de pesquisa no diretório do CNPq (base junho 2013). Foram realizadas 33 entrevistas com representantes dos 34 grupos de pesquisa do Instituto Butantan, pois um pesquisador era líder de dois grupos simultaneamente. Para as entrevistas, realizadas em 2014, foi utilizado um roteiro semiestruturado onde constavam questões sobre fontes de financiamento para pesquisas utilizadas onde se explicitava o PPSUS como uma destas fontes:
"Com se constituem as linhas de pesquisa em seu grupo? Adotam as pré-existentes ou adotam uma nova agenda de pesquisas? Como a agenda externa influi na pesquisa? As pautas e os problemas da área da saúde pública brasileira [como editais e linhas de financiamento] influenciam sua atuação como pesquisador? A demanda do setor saúde define linhas de pesquisa? E o PPSUS?”.

No que concerne aos aspectos éticos, os participantes da entrevista foram informados previamente sobre os objetivos do projeto e foi garantido o sigilo e confidencialidade dos dados. A seguir, foi solicitada a concessão de consentimento na participação na pesquisa. Os entrevistados receberam o roteiro previamente ao agendamento da entrevista, que foram gravadas e posteriormente transcritas. Para a análise foi utilizada a técnica de análise de conteúdo ${ }^{11}$. Os trechos das entrevistas aqui citados serão referidas como E1 a E6.

\section{RESULTADOS}

No período de 2004 a 2012, foram lançados cinco editais do PPSUS, operacionalizados pela Fundação de Amparo à Pesquisa do Estado de São Paulo (FAPESP) e 205 projetos foram financiados.

Ao se analisar esse conjunto levando-se em conta o destino do recurso pode-se perceber que 138 projetos (67\%) financiados pelo PPSUS nesse período estavam vinculados às universidades públicas. Num segundo bloco aparecem as instituições e unidades ligadas à Secretaria de Estado da Saúde, que tiveram 43 projetos financiados, $21 \%$ do total. Num outro patamar aparecem as universidades privadas, os serviços privados de saúde e as secretarias municipais de saúde com respectivamente 12, 10 e 02 projetos aprovados nesse período (Tabela 1).

Verifica-se durante este período um aumento de $13 \%$ para $27 \%$ na participação das unidades e institutos da SES-SP nos projetos financiados (Tabela 2). Dentre as instituições da SES destacaram-se o Instituto Adolfo Lutz (23\%), Hospital das Clínicas (16\%) e Instituto do Coração (14\%). Mesmo não aparecendo oficialmente 
como instituição proponente, foi possível identificar o Instituto Butantan como uma das instituições contempladas por financiamento do PPSUS, na medida em que o pesquisador principal, responsável por três projetos aprovados no período, é uma liderança científica reconhecidamente pertencente ao quadro do Instituto (Tabela 3).

Neste período foi aplicado por meio do PPSUS no estado de São Paulo o total de R $\$ 34.120 .000,00$ para o financiamento de projetos de pesquisa, onde as instâncias federal e estadual participaram paritariamente na composição orçamentária dessa linha de financiamento ${ }^{5}$.

As definições das prioridades em pesquisa em saúde para os editais PPSUS-SP passaram por diferentes formatos, ocasiões em que várias metodologias de construção e participação foram adotadas pelo comitê gestor do PPSUS8. De uma forma sucinta, os principais eixos e/ou temas-problema por ano do edital e respectivo processo de definição foram as seguintes:

i) Edital 2004-2005 - foram propostos dois eixos: 1) Qualificação da Atenção à Saúde QUALISUS; 2) Gestão Descentralizada do SUS. A elaboração desse edital foi realizada de forma centralizada pelo gabinete central da secretaria da saúde;

ii) Edital 2006-2007 - apresenta duas áreas temáticas: 1) Sistemas e Serviços de Saúde: Gestão do Trabalho- Epidemiologia e Informação em Saúde- Organização e Gestão e 2) Programas, Práticas e Ações de Saúde: Doenças Transmissíveis - Doenças não Transmissíveis - Ações de Saúde - Ações para Grupos Populacionais-Acesso a Medicamentos e insumos - Níveis, modalidades, protocolos e procedimentos. A construção das prioridades foi definida por um comitê gestor do PPSUSSP, utilizando duas rodadas de questionários enviados aos gestores regionais e municipais de saúde; iii) Edital de 2008-2009 - apresentou seis temas/ problemas: 1) Doenças não transmissíveis; 2) Doenças transmissíveis; 3) Morbidade e mortalidade por causas externas; 4 ) Morbidade e mortalidade materna e infantil; 5) Promoção/ proteção da saúde e meio ambiente; 6) Gestão e gerência do SUS. O processo de priorização passou por uma ampla consulta envolvendo gestores, pesquisadores e membros da comunidade e do conselho estadual de saúde;

iv) Edital 2010-2011 - são apresentados nove temas/problemas: 1) Construção das Redes Regionalizadas de Atenção à Saúde (RRAS) no SUS-SP; 2) Regulação; 3) Acesso, adesão e uso racional aos medicamentos nos níveis de atenção básica e média complexidade; 4) Modelos de avaliação e gestão de tecnologias para as redes no contexto da Política Estadual de Ciência Tecnologia e Inovação em Saúde para fortalecimento das RRAS do SUS-SP; 5) Processos de formação e Educação Permanente em saúde para a qualificação das RRAS; 6) Tecnologias de vigilância e controle de agravos à saúde decorrentes da exposição a poluentes e/ou contaminantes ambientais: ar, solo, água e alimentos; 7) Doenças transmissíveis: avaliação da magnitude; diagnóstico e tratamento; resultados e impacto de práticas assistenciais; 8) Avaliação das estratégias, magnitude, métodos diagnósticos, resultados e impactos das práticas e procedimentos das Redes Temáticas de atenção à saúde; 9) Sustentabilidade do Financiamento do SUS-SP para as RRAS: modelos de financiamento de redes de atenção à saúde em sistemas universais e modelos de pagamento a prestadores e transferência de recursos. Este edital seguiu as recomendações do DECIT12, passando por três etapas de consulta, que incluíram gestores, pesquisadores e representantes dos conselhos de saúde seguida de uma oficina para definição de prioridades ${ }^{8}$; 
v) Edital 2012-2013 - apresentou cinco grandes eixos: 1) Redução da morbimortalidade por doenças e agravos não transmissíveis; 2) Controle de doenças transmissíveis; 3) Gestão do SUS e Redes Regionais de Atenção à Saúde; 4) Implementação de Políticas/Programas Prioritários; 5) Incorporação/desincorporação de tecnologias. A definição de prioridades foi feita tomando como o PES 2012-2015 e por meio da realização de oficinas de prioridades com participação de gestores, comunidade científica e controle social

A análise dos te $\neg$ mas/problemas apresentados nos cinco editais do PPSUS-SP lançados no período, indica uma forte tendência para assuntos relacionados à assistência, gestão de programas ou estratégias de saúde. Ao cotejarmos esses temas às linhas de pesquisa do Instituto Butantan, considerando seu perfil histórico de atuação, com exceção do edital de 2005 , verificou-se a presença de pautas cujos grupos de pesquisa do Instituto Butantan teriam oportunidade de aplicar projetos.

Ao analisarmos os temas da agenda, levando-se em consideração os critérios da ANPPS identificamos, para cada projeto financiado pelo PPSUS-SP, qual item da sub-agenda está relacionado. Nessa análise, verificase que $32 \%$ dos projetos financiados pertencem a três itens da sub-agenda: "Complexo Industrial da Saúde", "Doenças transmissíveis" e "Pesquisa clínica". Nesse sentido, é possível dizer que em pelo menos nesses três itens dessa Agenda há compatibilidade com as linhas de pesquisa historicamente desenvolvidas pelo Butantan (Tabela 4). Durante o período estudado, o Butantan efetivamente participa com três projetos, nomeadamente: "Produção de lotes experimentais de vacina contra Dengue" e "Desenvolvimento industrial de surfactante pulmonar de origem animal" no ano de 2004-2005, e "Hepatite B - novas medidas preventivas e terapêuticas” financiado em 2006-2007.

O Instituto Butantan é uma instituição já consolidada na plataforma de pesquisa nacional, possui um quadro qualificado de pesquisadores de carreira e durante sua trajetória institucional já contou com diversos grupos de pesquisa de referência no cenário estadual e nacional. A partir de 1992, com a instituição dos diretórios de grupos de pesquisa do $\mathrm{CNPq}$, os grupos de pesquisa da instituição, alguns deles com trajetórias que remontam à década de 1980, foram cadastrados na Plataforma Lattes. Atualmente, o Butantan possui um quadro de 173 pesquisadores de carreira, dos quais $80 \%$ possuem doutorado ou pós-doutorado. Além disso, associada à atividade de pesquisa e produção de imunobiológicos, o Butantan é um importante órgão de formação científica, oferecendo cursos de divulgação científica, extensão universitária, estágios e programas de pós-graduação lato sensu e stricto sensu. Além disso, é oferecido um programa de mestrado e doutorado em biotecnologia em parceria com o Instituto de Biociências da Universidade de São Paulo (ICB-USP) e os laboratórios da instituição são campo de pesquisa de alunos oriundos de diversos programas de pós-graduação, o que reforça o papel histórico da instituição no que tange à formação de recursos humanos.

Os trinta e quatro grupos de pesquisa do Butantan cadastrados no diretório do CNPq até 2013 abrangem nove áreas do conhecimento direta ou indiretamente relacionadas com a saúde pública, doenças transmissíveis e imunobiológicos. A instituição apresentou nas últimas décadas um considerável crescimento e diversificação destes grupos, com destaque para os grupos de farmacologia, imunologia, microbiologia e bioquímica (Tabela 5).

\section{DISCUSSÃo}

Verifica-se pelos dados coletados por meio das entrevistas uma forte identidade institucional e ênfase na pesquisa básica. Além disso, foi possível identificar quatro categorias distintas de líderes de grupos de pesquisa, no que se refere ao tema financiamento e o PPSUS. É importante mencionar que em uma entrevista o tema financiamento não foi 
considerado pelo entrevistado e, desta forma, foram analisadas 32 entrevistas que abordaram o tema.

A primeira categoria, composta por 21 pesquisadores, ignoraram o PPSUS como uma fonte de financiamento, não citando o programa em suas considerações, exemplificado por uma fala, característica, de um integrante deste grupo:

E os editais, a gente sempre vai atrás; a gente está sempre alerta; quando aparece alguma oportunidade a gente sempre pede. Tudo o que nós temos de equipamento a gente conseguiu com verba de fora - é FAPESP, é CNPq, a gente vai para a CAPES, convênios internacionais, todo mundo já teve auxílio aqui de pesquisa. Então a gente sempre tem, auxílios em vigência e temos certa independência (E1).

Ainda nesta categoria, verificamos em muitos líderes de grupo uma postura negativa com relação a editais ou processos de indução de temas de pesquisa:

...os editais não influenciam a minha pesquisa. Eu posso ter uma pesquisa, uma pergunta que se ajuste àquele edital..., mas eu não ajusto a minha pesquisa; nunca consegui fazer isso. Mesmo porque [...] O envenenamento como doença negligenciada, ele é absolutamente negligenciado no Brasil! Apesar do envenenamento ser um problema de saúde pública neste país! Então, editais sobre isso eu nunca vi [...], mas eu não tenho nenhum que tenha abraçado em função disso; nenhum. Para mim, não influencia o meu caminho, nem do meu grupo (E2).

A segunda categoria identificada refere-se a três pesquisadores que, apesar de reforçar a ideia de que a pesquisa desenvolvida pelo grupo sob sua liderança é relacionada às questões de saúde pública, desconhecem o PPSUS como uma possibilidade de financiamento de pesquisa. Falas como as seguintes ilustram esse entendimento:

Linha de pesquisa? E o que é o PPSUS?[...] não, fui incluída exatamente nesse programa, mas o que temos feito sempre visou a Saúde Pública (E3).
A terceira categoria inclui sete pesquisadores que conhecem o programa, mas afirmam que os editais não são compatíveis com as linhas de pesquisa desenvolvidas pelo grupo ou ainda afirmam que não se identificam com as propostas dos editais:

A gente tem respondido demandas que estão aí. Agora, em relação ao PPSUS eu acho que a gente, por fazer pesquisa básica, a distância é muito grande. Então, quando eu vejo edital do PPSUS eu até olho, mas não [...] não tem assim um encaixe imediato, não. Seriam mais editais [...] Agora a gente está entrando com um projeto junto ao Biota ... outras políticas públicas com que a gente contribui. Mas com o PPSUS mesmo, eu não vejo muita [...] (E4).

Na realidade eu nunca solicitei. Porque essa minha linha entraria plenamente - a parte de diagnóstico - dentro do PPSUS. Eu acabei nunca aplicando ao PPSUS; isso eu nunca apliquei [...] conheço editais e acho que, pelo que eu leio sempre, acho que coincidentemente as vezes que houve essa chamada eu já tinha projeto em andamento; $e$ agora, com essas regras que você não pode ter dois projetos e tal..., mas nunca foi uma demanda do Instituto de eu fazer diagnóstico, o Butantan tem algumas demandas em algumas áreas, mas essa de diagnóstico não era uma demanda (E5).

A quarta e última categoria é constituída por um único líder de grupo que conhece, entende que este programa tem relevância para o SUS e já participou de projetos financiados pelo PPSUS, embora em parceria com outra instituição coordenadora do projeto:

Os grupos têm pouca produção em Saúde Coletiva neste instituto. Veja o PPSUS, excelente, já participamos de dois PPSUS (E6).

Efetivamente, é possível dizer que nenhum dos entrevistados utilizou-se institucionalmente do financiamento PPSUS. Das fontes de financiamento, a FAPESP* aparece largamente como a mais citada, seguida do CNPq, Financiadora de Estudos e Projetos (FINEP) e outras em proporção reduzida o

\footnotetext{
* - Lançado em março de 1999, o objetivo do Programa FAPESP de Pesquisas em Caracterização, Conservação, Restauração e Uso Sustentável da Biodiversidade (BIOTA-FAPESP) é conhecer, mapear e analisar a biodiversidade do Estado de São Paulo.
} 
que permite verificar, portanto, uma baixa adesão ou mesmo desconhecimento destes pesquisadores na agenda de pesquisa do SUS.

No período estudado constata-se um resultado auspicioso na implantação DO Programa de Pesquisa para o SUS no Estado de São Paulo13, na medida em que foram investidos em pesquisa um montante de 34 milhões de reais, colocou-o na $2^{2}$ posição no contexto nacional no que tange ao volume de recursos investidos. No que diz respeito às instituições que receberam recursos do programa, é possível dizer que houve uma participação relevante das unidades da SES-SP atingindo, em 2011, mais de um quarto dos projetos financiados no estado, situação única no país. Contudo, o Instituto Butantan teve uma participação relativamente modesta frente à sua tradição e estrutura instalada de pesquisa.

Analisando comparativamente estudos realizados em outras unidades federadas, verificamos alguns obstáculos que constituem lugar comum entre diversos estados brasileiros na implementação do PPSUS, semelhante ao que ocorre em São Paulo: dificuldades no processo de escolha de prioridades em pesquisa; precária participação da sociedade civil e complexidade da incorporação dos resultados das pesquisas ${ }^{8,14,15,16,17,18}$. Na mesma análise, constatouse, levando em conta as agendas dos outros estado e a do PPSUS-SP, diversas oportunidades para desenvolvimento de projetos cujos temas contemplavam áreas em que os grupos de pesquisa do Instituto Butantan, pela sua tradição de pesquisa, teriam oportunidade de contribuir, tais como: doenças transmissíveis, diagnóstico e controle de doenças (soros e vacinas e temas correlacionados). A esse respeito, vale apontar como caso emblemático um dos grupos de pesquisa investigado que aponta como uma de suas linhas de pesquisa no diretório de grupos de pesquisa do CNPq, "diagnóstico de doenças transmissíveis e desenvolvimento tecnológico" que poderia em tese ajustar-se perfeitamente às propostas do PPSUS-SP. No que diz respeito a patentes, área em que o Instituto Butantan tem uma atuação relevante, destacamos dois estados, Alagoas e Rio Grande do Sul, que apresentaram pedidos de patentes, como resultado de projetos financiados pelo PPSUS ${ }^{16,17}$.

Chama atenção o caso dos projetos que aparecem como diretamente ligados à SES-SP e que foram propostos e conduzidos por uma liderança científica vinculada ao Instituto Butantan. Contemplados em 2005 nos eixos temáticos do Edital "Qualificação da Atenção à Saúde - QUALISUS" e "Gestão Descentralizada do SUS”, aparentemente não seriam compatíveis com as linhas de pesquisa tradicionalmente desenvolvidas no Instituto. Adicionalmente e de maneira incoerente, nos anos subsequentes os editais que contemplavam temas relacionados às linhas de pesquisa dos diversos grupos de pesquisa do Instituto, não se verificou projetos financiados.

Contudo, a análise comparativa com outros estados não ajuda a explicar a não adesão de um instituto como o Butantan, que atende às demandas de saúde pública desde sua fundação, baseada apenas na conjuntura do PPSUS. Uma particularidade dos grupos de pesquisa que pareceu relevante para interpretação dos resultados consistiu na declaração explícita, da maioria absoluta dos líderes de grupo, de sua orientação para pesquisa básica. De um modo geral, os pesquisadores manifestam-se avessos à pesquisa induzida, afirmando preferir a submissão de projetos às agências de fomento por meio do fluxo contínuo, especificamente na modalidade "auxílio regular à pesquisa" da FAPESP.

Outra explicação poderia recair sobre os valores financiados. No entanto, observa-se que o valor médio dos projetos financiados pelo PPSUS no período estudado foi de aproximadamente $R \$ 166.000,00$, enquanto que se verifica que entre 2006 a 2016 os projetos financiados pela FAPESP (modalidade auxílio regular à pesquisa) vinculados ao Butantan tiveram um valor médio de $\mathrm{R} \$ 93.538,1519$. Portanto, pode-se afirmar novamente, que o descompasso 
existente entre os projetos institucionais e a não percepção do PPSUS-SP como uma possibilidade de financiamento não se justifica em função dos valores de solicitados e recebidos.

Outras hipóteses possíveis para explicar a reduzida participação do Instituto nestes cinco editais do PPSUS podem recair em três aspectos verificados pelas entrevistas: a) Ausência de divulgação institucional, e, em um sentido mais amplo, de entendimento de um compromisso institucional com o SUS; b) Aspectos da cultura institucional - trajetória dependente e tradição das linhas de pesquisa já historicamente consolidadas, ligadas ao que o líderes entrevistados definem como "pesquisa básica”; c) Conjuntura conveniente, propiciada pelo financiamento da FAPESP, associada a uma postura reativa com relação à pesquisa induzida; d) Visão de que o PPSUS se restringe à prestação de serviços e de atendimento.

Outro aspecto relevante é a constatação, atualmente no país, da existência de uma cultura, não apenas institucional, mas também acadêmica, de indução à pesquisa, que preza mais por resultados de curto prazo e de maior aplicação direta de novas tecnologias e serviços que acaba por impor mudanças ao novo cenário de pesquisa em saúde pública. Devese considerar entretanto que na perspectiva das pesquisas da área biomédica - que demanda estrutura laboratorial complexa; desenvolvimento de modelos experimentais envolvendo biotérios que demandam um cuidado especial com relação aos aspectos éticos de pesquisa com animais; equipamentos específicos de alto custo. A adaptação destes grupos pesquisa às propostas para atender demandas mais imediatas do Sistema Único de Saúde com respectivas respostas, envolvem riscos, tanto no que concerne a possibilidades de resultados concretos quanto, e não menos importante, ao desempenho científico e produtividade do líder e seu grupo de pesquisa.

Os resultados da investigação aqui apresentados indicam aspectos importantes dessa política pública que devem ser reexaminados quanto ao modelo PPSUS adotado no Estado de São Paulo, considerando o princípio da execução descentralizada e o modelo participativo para eleição das prioridades.

A partir desses resultados, algumas questões podem ser levantadas: que tipo de pesquisa os gestores da Secretaria de Saúde entendem que o PPSUS deve atender? A esse respeito, Chaimovich ${ }^{20}$ destaca "o delicado equilíbrio entre a liberdade dos cientistas e a necessidade social do conhecimento". Ainda, como considerar o perfil de uma instituição como o Butantan, seus grupos de pesquisa, laboratórios e suas especificidades na priorização dos problemas de saúde a serem investigados? No que tange ao contexto interno ao Butantan, como lidar com a cultura institucional? O que explica um líder de grupo de pesquisa do Butantan identificar sua linha de pesquisa com um projeto que visa a caracterização, conservação, restauração e uso sustentável da biodiversidade e não com o PPSUS? Seria isto um indicativo da necessidade de uma maior aproximação do Instituto com os demais órgãos da SES-SP?

\section{CONCLUSÕES}

Efetivamente, é possível dizer que entre os objetivos idealizados para o PPSUS estão a redução das desigualdades regionais, o fortalecimento da capacidade de pesquisa dos estados e o apoio ao desenvolvimento das pesquisas de acordo com as demandas locais de serviços de saúde - apenas este último aplica-se ao caso de São Paulo. Contudo, este deve ser relativizado considerando o estágio atual de desenvolvimento do parque de C\&T instalado no Estado. A questão do direcionamento dos temas de pesquisa voltados para demandas prioritárias do SUS-SP seria a que permanece relevante e a ser investigada.

Ainda que as respostas demandem uma investigação mais aprofundada das causas e possíveis explicações, verifica-se que um esforço deve ser envidado por 
parte dos gestores para definição de temas que incorporem institutos com perfil como o Butantan. Paralelamente, caberia ao Instituto Butantan, como órgão pertencente ao Sistema Único de Saúde, criar estratégias institucionais de aproximação de seus grupos de pesquisa às prioridades de saúde apontadas no PPSUS. Uma conduta saudável seria a revisão do formato dos editais, concomitantemente à adoção de um programa institucional intensivo de difusão de informações e discussões com o corpo de pesquisadores a respeito do Sistema de Saúde Brasileiro, na perspectiva de que este possa dispor do potencial cientifico e tecnológico que representa o Instituto Butantan.

\section{REFERÊNCIAS}

1. Guimarães R. Pesquisa em saúde no Brasil: contexto e desafios. Rev. Saúde Públ. [Internet]. 2006 Aug [cited 2018 June 19]; 40(spe): 3-10. Available from: http:// www.scielo.br/scielo.php?script=sci_arttext\&pid=S003489102006000400002\&lng=en. http://dx.doi.org/10.1590/S003489102006000400002 .

2. Ministério da Saúde (BR). Secretaria de Ciência, Tecnologia e Insumos Estratégicos. Departamento de Ciência e Tecnologia. Por que pesquisa em saúde? (Série B. Textos Básicos de Saúde). [Internet]. 2007 [cited 2017 nov 18]. Brasília. 1-20, Available from: http://bvsms.saude.gov.br/bvs/publicacoes/pesquisa saude.pdf .

3. Ministério da Saúde (BR). Secretaria de Ciência, Tecnologia e Insumos Estratégicos. Departamento de Ciência e Tecnologia. Programa de fomento à pesquisa para os sistemas locais de saúde: gestão compartilhada em saúde. 2006. Rev. Saúde Pública, 40 (6), 1131-1136.

4. Ministério da Saúde (BR). Secretaria de Ciência Tecnologia e Insumos Estratégicos. Departamento de Ciência e Tecnologia. Descentralização no contexto da pesquisa em saúde. 2011. Rev. Saúde Pública, 45 (3), 626-630.

5. Ministério da Saúde (BR). Panorama situacional do PPSUS 2014. [Internet]. Available from: http://portalsaude.saude.gov.br/ index.php/o-ministerio/principal/leia-mais-o-ministerio/363sctie-raiz/decit-raiz/ppsus/12-ppsus/10533-ppsus-estados [Cited: 2017 nov 18]

6. Secretaria de Estado da Saúde (SP). Plano Estadual de Saúde 2008-2011. [Internet]. Available from: http://www.saude. sp.gov.br/resources/ses/perfil/gestor/normas-e-procedimentos/ plano_estadual_de_saude_2008_2011.pdf . [Cited: 2017 nov 18].

7. Secretaria de Estado da Saúde (SP). Plano Estadual de Saúde 2012-2015. [Internet]. Available from: http://www. saude.sp.gov.br/resources/ses/perfil/gestor/documentos-de- planejamento-em-saude/plano-estadual-de-saude-2012-2015sessp/pes_2012_2015.pdf [Cited 2017 nov 17].

8. Toma TS, Bersusa AAS, Martino LVS, Venâncio SI. PPSUS e a definição de prioridades de pesquisa para os editais de 2004 a 2012 no estado de São Paulo. BIS, Bol. Inst. Saúde (Impr.) [Internet]. 2011 Abr [cited 2017 Jun 20]; 13(1): 76-84. Available from: http://periodicos.ses.sp.bvs.br/scielo.php?script=sci_ arttext\&pid=S1518-18122011000100012\&lng=pt.

9. Creswell JW, Clark VLP. Pesquisa de métodos mistos. $2^{\circ}$ Ed. Porto Alegre: Editora Penso, 2013.

10. Fundação de Amparo à Pesquisa do Estado de São Paulo (FAPESP), Sistema AGILIS - base pública de consulta WEB. [Internet]. Available from: http://internet.aquila.fapesp.br/agilis/ publico/ and http://www.fapesp.br/4819 [cited 2017 nov 15].

11. Bardin L. Análise de conteúdo. 4ª Ed. Lisboa: Edições 70. 2009.

12. Ministério da Saúde (BR). Departamento de Ciência e Tecnologia. Seleção de prioridades de pesquisa em saúde: guia PPSUS. (Série A. Normas e Manuais Técnicos). [Internet]. 2008 [cited 2017 nov 15]. Brasília. 1-74. Available from: http://bvsms. saude.gov.br/bvs/publicacoes/selecao_prioridades_pesquisa_ saude_ppsus.pdf .

13. Secretaria de Estado da Saúde (SP). Instituto de Saúde. PPSUS-SP: Experiências exitosas. [Internet] 2015 [cited 2017 nov 18]. Available from: https://madmagz.com/ magazine/614158\#/page/33.

14. Ell E, Batista CJ, Santos Junior JE, Barbosa Junior A, Frattini NAC, Sachetti CG et al. Programa Pesquisa para o SUS (PPSUS): contribuições para a ciência, tecnologia e inovação em saúde no estado do Paraná. Espaç. Saúde. [Internet] 2016. [ cited 2017 nov 5] 17 (1). 65-74. Available from: http://dx.doi. org/10.22421/1517-7130.2016v17n1p65 .

15. Molesini, JA, Formigli VLA, Guimarães MCL, Melo CMM. Programação pactuada integrada e gestão compartilhada do SUS. Rev. Baiana de Saúde Pública 2010; 34(3):623-638.

16. Amaral NM, Khalili JB, Camerino MBC, Melo MCO, Menezes VM, Santos MGS. A gestão das pesquisas para o SUS em Alagoas. BIS, Bol. Inst. Saúde (Impr.) [Internet]. 2011 Abr [cited 2018 Jun 19]; 13(1): 85-91. Available from: http://periodicos.ses.sp.bvs.br/scielo.php?script=sci arttext\&pid=S1518-18122011000100013\&lng=pt .

17. Guidini MB, Calabró L, Mello RL, Pereira ES, Souza DOG. Avaliação de Resultado do Programa de Pesquisa para o SUS no RS. In: Anais do X Encontro Nacional de Pesquisa em Educação em Ciências. Associação Brasileira de Pesquisa em Educação em Ciências. [internet] 2015 nov : 24-27 [cited 2017 nov 18]. Águas de Lindóia. SP. Brasil. Available from: http://www.abrapecnet.org.br/enpec/x-enpec/anais2015/ listaresumos.htm

18. Carvalho RRS, Jorge MSB, Serapioni M, Morais JB, Caminha ECCR. Programa Pesquisa para o SUS: desafios para aplicabilidade na gestão e serviços de saúde do Ceará. Saúde debate [Internet]. 2016 Sep. [cited 2018 June 19]; 40(110): 5363. Available from: http://www.scielo.br/scielo.php?script=sci_ 
arttext\&pid=S0103-11042016000300053\&lng=en . http://dx.doi. org/10.1590/0103-1104201611004.

19. Fundação de Amparo à Pesquisa do Estado de São Paulo (FAPESP). Relatórios síntese de 2006 a 2012[Internet]. Available from: http://www.fapesp.br/publicacoes [cited 2017 nov. 12].
20. Chaimovich H. Brasil, ciência, tecnologia: alguns dilemas e desafios. Estud. av. [internet]. 2000,14 (40): 134-143 [cited 201706 20], Available from: http://www.scielo.br/scielo. php?script=sci_arttext\&pid=S0103-40142000000300014\&lng=e n\&nrm=iso >. ISSN 0103-4014. http://dx.doi.org/10.1590/S010340142000000300014

\section{FIGURAS E TABELAS}

Tabela 1 - Distribuição e percentual de projetos financiados pelo PPSUS-SP no período de 2004 à 2012

\begin{tabular}{|lccccccc}
\hline Edital-ano & & & & & & \\
TIPOS DE INSTITUIÇÃO & $\mathbf{2 0 0 4}$ & $\mathbf{2 0 0 6}$ & $\mathbf{2 0 0 8}$ & $\mathbf{2 0 1 0}$ & $\mathbf{2 0 1 2}$ & TOTAL & PERCENTUAL \\
\hline Unidades da SES-SP & 4 & 10 & 9 & 11 & 9 & 43 & $21 \%$ \\
Universidades Públicas & 26 & 36 & 24 & 24 & 25 & 138 & $67 \%$ \\
Universidades Privadas & 1 & 5 & 2 & 3 & 1 & 12 & $6 \%$ \\
Secretarias Municipais de & 0 & 5 & 0 & 1 & 0 & 2 & $1 \%$ \\
Saúde & & & & & & & \\
Serviços privados & 0 & 5 & 2 & 2 & 1 & 10 & $5 \%$ \\
TOTAL & 31 & 60 & 37 & 41 & 36 & 205 & $100,00 \%$ \\
& & & & & & & Fonte: FAPESP
\end{tabular}

Tabela 2 - Proporção de projetos aprovados por tipo de instituição e ano do edital, PPSUS-SP

\begin{tabular}{lccccc} 
TIPOS DE INSTITUIÇÃO & $\mathbf{2 0 0 4}$ & $\mathbf{2 0 0 6}$ & $\mathbf{2 0 0 8}$ & $\mathbf{2 0 1 1}$ & $\mathbf{2 0 1 2}$ \\
\hline Unidades da SES-SP & $13 \%$ & $17 \%$ & $24 \%$ & $27 \%$ & $25 \%$ \\
Universidades Públicas & $84 \%$ & $65 \%$ & $65 \%$ & $59 \%$ & $69 \%$ \\
Universidades Privadas & $3 \%$ & $8 \%$ & $5 \%$ & $7 \%$ & $3 \%$ \\
Secretarias Municipais de & $0 \%$ & $2 \%$ & $0 \%$ & $2 \%$ & $0 \%$ \\
Saúde & & & & & \\
Serviços privados & $0 \%$ & $8 \%$ & $5 \%$ & $5 \%$ & $3 \%$ \\
TOTAL & $100 \%$ & $100 \%$ & $100 \%$ & $100 \%$ & $100 \%$ \\
\end{tabular}


Tabela 3 - Número e percentual de projetos de unidades da SES-SP financiados pelo PPSUS-SP no período de 2004 à 2012

\begin{tabular}{lccccccc} 
INSTITUIÇÕES DA SES-SP & $\mathbf{2 0 0 4}$ & $\mathbf{2 0 0 6}$ & $\mathbf{2 0 0 8}$ & $\mathbf{2 0 1 1}$ & $\mathbf{2 0 1 2}$ & TOTAL & $\%$ \\
\hline Inst. Adolfo Lutz & 0 & 1 & 3 & 5 & 1 & 10 & 23 \\
Hosp. Clínica São Paulo & 0 & 4 & 0 & 1 & 2 & 7 & 16 \\
Inst. Coração do Hosp. Clínica São Paulo & 1 & 1 & 2 & 1 & 1 & 6 & 14 \\
Hop. Clínicas Ribeirão Preto & 0 & 0 & 0 & 2 & 2 & 4 & 9 \\
Inst. Saúde & 1 & 0 & 2 & 0 & 1 & 4 & 9 \\
Secretaria da Saúde de São Paulo* & 2 & 1 & 0 & 0 & 0 & 3 & 7 \\
Fund. Pro Sangue Hemocentro São Paulo & 0 & 1 & 0 & 1 & 1 & 3 & 7 \\
Superintendência Controle Endemias & 0 & 0 & 1 & 0 & 1 & 2 & 5 \\
Fac. Medicina Marilia & 0 & 2 & 0 & 0 & 0 & 2 & 5 \\
Fund. Hemocentro Ribeirão Preto & 0 & 0 & 0 & 1 & 0 & 1 & 2 \\
Inst. Lauro Souza Lima & 0 & 0 & 1 & 0 & 0 & 1 & 2 \\
\hline TOTAL SES & 4 & 10 & 9 & 11 & 9 & 43 & 100 \\
\hline
\end{tabular}

Tabela 4 - Frequência de projetos aprovados por subárea da ANPP-MS, por ano de edital, PPSUS-SP

$\begin{array}{lccccccc}\text { SUB ÁREA } & \mathbf{2 0 0 4} & \mathbf{2 0 0 6} & \mathbf{2 0 0 8} & \mathbf{2 0 1 1} & \mathbf{2 0 1 2} & \text { TOTAL } & \text { \% } \\ \text { Doenças não transmissíveis } & 16 & 14 & 8 & 14 & 6 & 59 & 29 \\ \text { Completo produtivo da saúde } & 1 & 13 & 4 & 6 & 8 & 31 & 15 \\ \begin{array}{l}\text { desenvolvimento tecnológico) } \\ \text { Gestão do trabalho, educação e saúde }\end{array} & 7 & 7 & 6 & 2 & 7 & 29 & 15 \\ \text { Doenças transmissíveis } & 3 & 8 & 7 & 8 & 2 & 28 & 14 \\ \text { Sistemas e políticas de saúde } & 1 & 11 & 9 & 3 & 2 & 26 & 13 \\ \text { Promoção à saúde } & 1 & 0 & 2 & 0 & 6 & 9 & 4 \\ \text { Epidemiologia } & 0 & 1 & 0 & 4 & 4 & 9 & 4 \\ \text { Pesquisa clínica } & 0 & 4 & 0 & 3 & 0 & 7 & 3 \\ \text { Saúde bucal } & 2 & 2 & 0 & 0 & 1 & 5 & 2 \\ \text { Saúde Mental } & 0 & 0 & 1 & 1 & 0 & 2 & 1\end{array}$

Fonte: FAPESP. 
Tabela 5 - Grupos de pesquisa cadastrados no CNPq, por área e por ano de criação - Instituto Butantan ano base 2013

\begin{tabular}{|c|c|c|c|c|c|c|c|c|c|c|c|c|c|c|c|c|c|}
\hline \multirow[b]{2}{*}{ ÁREA } & \multicolumn{17}{|c|}{ ANO } \\
\hline & 1980 & 1985 & 1988 & 1993 & 1994 & 1995 & 1997 & 2000 & 2001 & 2002 & 2004 & 2005 & 2006 & 2008 & 2010 & 2012 & 2013 \\
\hline Farmacologia & 1 & 1 & 1 & 2 & 2 & 2 & 2 & 3 & 3 & 4 & 4 & 5 & 5 & 5 & 5 & 5 & 5 \\
\hline Imunologia & - & 1 & 2 & 2 & 2 & 3 & 3 & 3 & 3 & 4 & 4 & 4 & 4 & 6 & 7 & 7 & 7 \\
\hline Microbiologia & - & - & - & - & 1 & 1 & 1 & 1 & 1 & 2 & 2 & 3 & 5 & 5 & 5 & 5 & 5 \\
\hline Bioquímica & - & - & - & - & - & 1 & 1 & 1 & 2 & 5 & 5 & 5 & 5 & 5 & 5 & 5 & 7 \\
\hline Morfologia & - & - & - & - & - & - & 1 & 1 & 1 & 1 & 1 & 1 & 1 & 1 & 1 & 1 & 1 \\
\hline Saúde Coletiva & - & - & - & - & - & - & - & - & - & 1 & 1 & 2 & 2 & 2 & 2 & 2 & 2 \\
\hline Zoologia & - & - & - & - & - & - & - & - & - & - & 1 & 2 & 2 & 2 & 2 & 4 & 4 \\
\hline Genética & - & - & - & - & - & - & - & - & - & - & - & - & 1 & 1 & 1 & 1 & 1 \\
\hline Parasitologia & - & - & - & - & - & - & - & - & - & - & - & - & - & 1 & 1 & 1 & 1 \\
\hline Total & 1 & 2 & 3 & 4 & 5 & 7 & 8 & 9 & 10 & 17 & 18 & 22 & 25 & 28 & 29 & 31 & 33 \\
\hline
\end{tabular}

